\title{
Toward Acceptable Domestic Robots: Applying Insights from Social Psychology
}

\author{
James E. Young - Richard Hawkins - Ehud Sharlin - Takeo Igarashi
}

Received: date / Accepted: date

\begin{abstract}
Social psychology offers a perspective on the acceptance and adoption of technology that is not often considered in technical circles. In this paper, we discuss several adoption-of-technology models with respect to the acceptance of domestic robots: we examine socialpsychology literature and apply it directly to humanrobot interaction. We raise key points that we feel will be pivotal to how domestic users respond to robots, and provide a set of guidelines that roboticists and designers of robotic interfaces can use to consider and analyze their designs. Ultimately, understanding how users respond to robots and the reasons behind their responses will enable designers to creating domestic robots that are accepted into homes.
\end{abstract}

CR Subject Classification H.1.2 [Models and principles]: user/machine systems-software psychology

\section{Introduction}

Over the last 25 years robots have permeated many application areas and industrial processes: consider, for

\section{James E. Young}

University of Calgary, Canada, JST ERATO, Japan

E-mail: jim.young@ucalgary.ca

Richard Hawkins

University of Calgary, Canada

Professor and Canada Research Chair in Science Technology and Innovation Policy

E-mail: rhawkins@ucalgary.ca

Ehud Sharlin

University of Calgary, Canada

E-mail: ehud@cpsc.ucalgary.ca

Takeo Igarashi

The University of Tokyo, Japan, JST ERATO, Japan

E-mail: takeo@acm.org example, robots in medical, military and public safety contexts. However, robots are only now starting to be introduced into the domestic environment as consumer products, entering into the everyday experience of individuals and families in their homes and communities. From robotic vacuum cleaners in millions of homes to robo-receptionists in Japanese offices, to medicinecarrying robots in hospitals, robots are poised to become a part of everyday life for the general public. Similar to how we encounter computing in our daily lives people may soon have little choice in the matter of interacting with robots, a movement that presents intractable challenges for both users and roboticists.

As robots start to enter homes, a key question for roboticists is 'What are the key dynamics and factors that influence how people perceive, understand, and ultimately accept robots?'. Certainly there is no question about their utility in the domestic context, especially in an era when more consumers seek relief from the day-today chores that eat into ever-scarcer leisure time. There is no question either of the immense consumer appetite for electronics goods in a huge variety of leisure markets. Nevertheless, most robots still exist in forms more appropriate for industrial applications. Also, there is an issue of cost as few household robots are available at mass-market consumer prices. However, these factors were common to virtually all advanced technologies that have been previously integrated into domestic contexts. In this paper, we propose that robots present several unique dynamics that differ substantially from previous 'domesticated' advanced technologies, and that robot designers and producers must consider these differences systematically in ways that go beyond many existing ideas about technology acceptance.

The issue of domestic robots illustrates a longstanding debate in the exploration of innovation phenom- 
ena, which revolves around the question of whether innovation is driven by demand or supply $[18,55]$. The problem is especially acute concerning heterogeneous consumer technology markets, which are far less well studied than industrial markets. Many contend that consumer markets in particular are not based solely on utility and price, but also by subjective calculations concerning social gains and functions $[36,61]$. The 'classical' Schumpeterian position is that demand plays little or no role at all; that innovation is directed entirely by entrepreneurs who force the development of new markets [53]. To the contrary, however, there is at least some empirical evidence of supply-demand interaction in industrial markets [52], although the role of consumer demand in innovation has remained much more obscure. It is becoming accepted, however, that innovation in consumer environments is highly dependent upon factors of socialization that merge utility with symbolic and cultural factors, and that this involves subtle transfers of knowledge from consumers to producers about emerging social trends and preferences $[41,65]$.

We argue that one of the most important and unique barriers to the widespread domestic adoption of robotics is an especially complex socialization process. The robotics environment is far more complex than most already established consumer technology markets, and the problems of technology acceptance are far more significant in a domestic environment than in an industrial one. By design, it is intended that domestic robots will enter into our personal spaces, where their mere physical presence will have an effect on the spaces they occupy $[13,68]$. Thus, the socialization of robots in the domestic context is far more than a conventional "human factors" design problem, in which barriers are overcome through the design of interfaces, infrastructures and routines. Neither is it merely a conventional "diffusion" problem whereby mass markets are created through positive feedback as more consumers experience and adopt a technology [51,60]. Instead, we argue that the domestic socialization of robots is largely dependent upon subjective consumer perceptions of what robots are, how they work and what exactly they are and are not capable of doing in a domestic environment. We argue also that understanding these elements requires that we understand them in the context of the social interactions, institutions and hierarchies into which domestic robots intervene.

In order to explore these arguments, we introduce several perspectives from social psychology, a branch of the social sciences that seeks to explain the relationships between individual perceptions and social behaviors. The economist Tibor Scitovski [54] was an early advocate of introducing this perspective to the problem of explaining the emergence of consumer demand; for example, suggesting that consumer satisfaction was related more to the psychological expectation of acquiring a product or service than to its actual acquisition and use. Subsequently, social psychology perspectives have been explored in more formal analytical frameworks for example, Montalvo [45] uses social psychology models to show analytically how decisions to innovate are conditioned by subjectively-defined 'willingness' factors. Our paper does not attempt to make a methodological contribution to social psychology. Rather, we borrow several concepts and analytical methods from social psychology in order to explore the role that subjectivity in consumer perceptions plays in influencing the socialization of domestic robots. By using these ideas to interpret recent domestic robot research and applications, we suggest how the insights gained from these comparisons might help re-conceptualize the design problem for domestic robots.

We start this paper by outlining the background to our work, specifying the particular problems that robots present in the domestic environment, along with some of the gaps in existing research. We next survey some relevant work in social psychology that deals generally with the domestication of technology, and then discuss how this work relates to the specifics of domestic robots. Finally, we distill the robot-specific social psychology analysis into a set of guidelines that developers and designers can use for analyzing and designing domestic robots.

\section{Background}

In this section we substantiate the problem of domesticating robotic technologies and outline how it relates to existing work. We introduce both the social psychology and human-robot interaction perspectives, and highlight how intersecting these perspectives with domestic robots yields new questions to be explored.

\subsection{Why Social Psychology?}

Most existing research into the adoption of robotic technology concerns the industrial application environment and generally focuses on financial, business, and economic concerns. This approach explores specific tasks and goal-oriented problems in terms of robotisability [22] (i. e., the ability to automate tasks with robots), general industrial automation issues (e.g., [21, $22,67]$ ), or macro and international-level industrial issues (e.g., [39]), but does not generally consider do- 


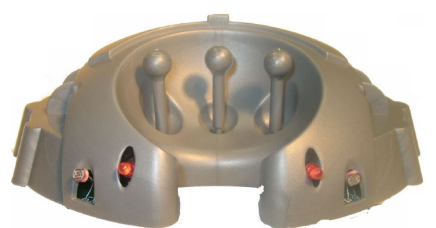

Fig. 1 A robot that Bartneck et. al. got users to kill. [6]

mestic social concerns (e.g., [59,65]). There are several conceptual models that address technology adoption in domestic contexts (such as $[2,19,40,63,62]$, discussed below). These consider user satisfaction, status and other technology socialization concerns. However, none of these address the special socialization characteristics and problems presented by robots.

Domestic robots are fundamentally different from other common domestic applications of advanced technology such as the ubiquitous PC. Robots have an invasive physical presence and a unique interface paradigm: they actively and physically share spaces with people and display a level of autonomy and intelligence. Unlike the PC, which stays where it is placed and must be actively engaged and enabled, a robot will physically interact with and alter its surroundings and may not remain in a simply-defined allocated space. Furthermore, unlike physically-safe PC-based virtual environments, interacting with a robot is more like interacting with a living entity. The robot may move unexpectedly, users must follow its motion cues and physical state, and may not have direct access to orthodox interfaces such as a keyboard or display panel. Thus, users of robotic technology often have to learn new interaction styles such as manipulation through remote control devices or voice commands [34]. This difference means that we cannot expect people to respond to robots in the same way that they do to other technologies. Much recent research shows that people respond to robots in unique ways, and often in ways similar to how they respond to living entities [5, 6, 8, 23, 24, 25, 26, 42]. For example, Bartneck et. al. explored how people negatively react to having to kill a robot (Figure 1). What this difference means for the acceptance of robots is as yet unclear, but it is at the heart of the question we explore in this paper.

One of the ways to understand how people perceive technology is to examine their reasons for adopting or not adopting it. Such examinations result in a better understanding of how much and in what fashion a person or household is willing or able to adopt a technology, as well how they are able to recognize the relevance or potential of new technologies as they appear - to interact with them and to learn to apply them in practical situations. In an industrial or organizational setting, this absorptive capacity is generally seen to be gener- ated by related knowledge - i. e. by existing capabilities upon which new capabilities can be built [16].

In trying to explain what determines the capacity of individuals and households to absorb domestic robots, however, we have to consider what constitutes relevant knowledge. From the perspective of an individual consumer, the understanding of technology is typically the result of social rather than scientific, technological or industrial activity $[7,13,15,67]$. Thus, the meaning of a technology is not limited to the mechanisms, physical and technical properties, or actual capabilities of the technology. Meaning extends also to how people think they must (or are supposed to) interact with technology and how it will (or should) integrate into and affect their lives.

Technology is defined largely by how people and the societies in which they live view, respond, and react to it. Thus, we can say that technology has a sociallyembedded meaning in the sense that how an individual shapes his or her understanding of a technology is directly linked to evolution in prevailing social attitudes. These attitudes, and the technology as well, do not necessarily move toward some idealistic or optimal goal, but rather are constructed from various complex and dynamic social relationships. This means of course that the number factors contributing to how people regard robots as social entities in everyday situations is astronomical, encompassing issues of culture, class, gender and age as well as social, political and economic structures and communication mechanisms $[20,51,56,57,70]$.

In this paper, as far as we know for the first time, we explore how some of the perspectives from social psychology can help us understand how domestic environments construct absorptive capacity with respect specifically to robots. We explicitly use this approach in order to shed additional light upon how the design of robots is related to human-robot interaction. Recognizing the enormous range of possible factors that could intervene in this process, we purposely limit our scope to just a few key factors and assume primarily the social context of contemporary North American culture.

\subsection{Human-Robot Interaction}

When a person interacts with a robot, there is a lack of common understanding which hinders communication; robots think in bits and bytes, a language that humans cannot inherently understand. When a robot enters a home, this general problem escalates, and the robot may clash with existing social structures.

A recent movement in the field of Human-Robot Interaction is the design of sociable robots, those which 
understand and communicate using human language to allow them to participate and be understood as social actors $[9,10,47]$ (for example, see Breazeal's Kismet, Figure 2). Sociable robots could use human-like facial expressions that indicate their general state, or gestures such as shrugging, indicating that they do not understand a command. Or they could monitor facial expressions to determine if users are happy or distressed. This approach, in addition to the pure utility of communication, also considers user comfort, perception, naturalness and ease of communication [9].

A potential danger with designing robots that mimic human social mechanisms is eeriness or creepiness (see Figure 3). Examples of robots that people find creepy are the $C B^{2}$ baby robot [44] (Figure 3(a)) or the Repliee R1 android [38]. Mori's uncanny valley [46] is one theory that tries to explain how certain robots can elicit a negative, uncanny, or eerie feeling in people. Generally, this theory proposes that likeness to a human can be directly related to familiarity, where the more human-like a robot is, the more believable and comfortable people find it. However, as likeness increases there is a breaking point beyond which familiarity drops and robots become eerie. This dropped level of comfort is called the uncanny valley (Figure 3(b)). Mori claims that this eeriness will not be overcome until robots mimic human sociality so well that we do not cue in on the fact that we are interacting with a robot.

The eeriness problem is of high interest to roboticists. The uncanny valley is just one model of this problem, one that is difficult to test and has little empirical evidence to back it up. Other research suggests more complex root and dimensions to the problem $[11,27,31$, $38,43,66]$, and some projects try to avoid eeriness by using mechanical designs $[32,33]$ or interfaces that use social principles without appearing human-like [69].

Recent research in sociable robotics discusses higher-level behavior patterns. Hamill and Harper [29,

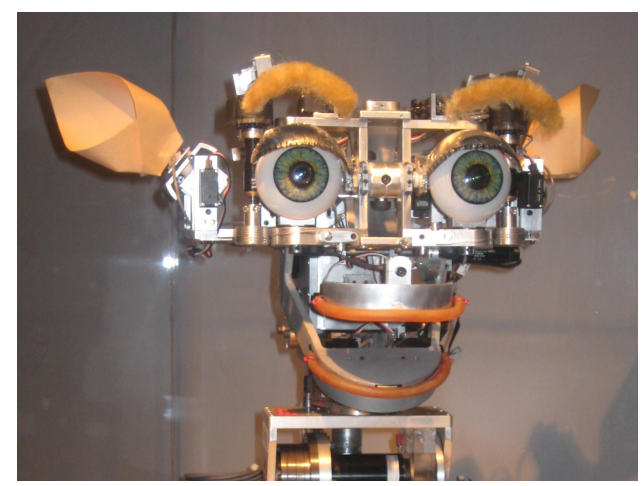

Fig. 2 Kismet, designed to explore the concepts of sociable robots. [10]

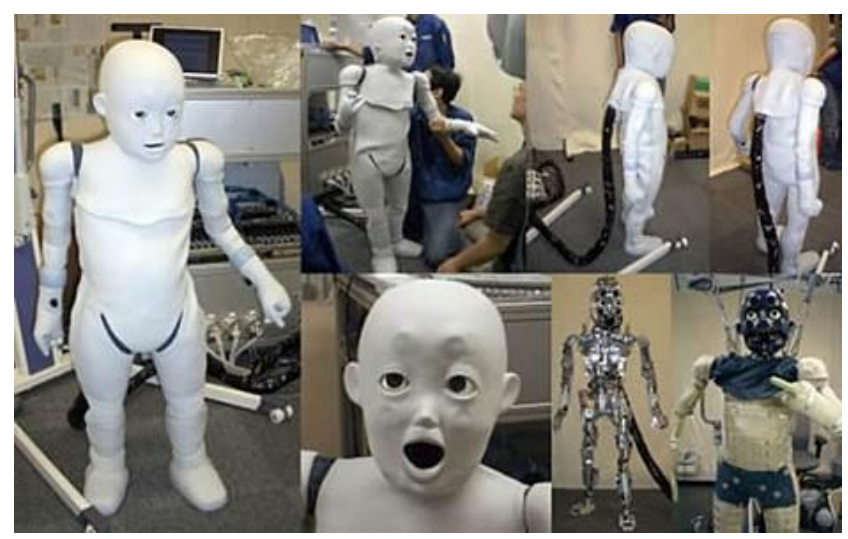

(a) $C B^{2}$ baby robot. [44]

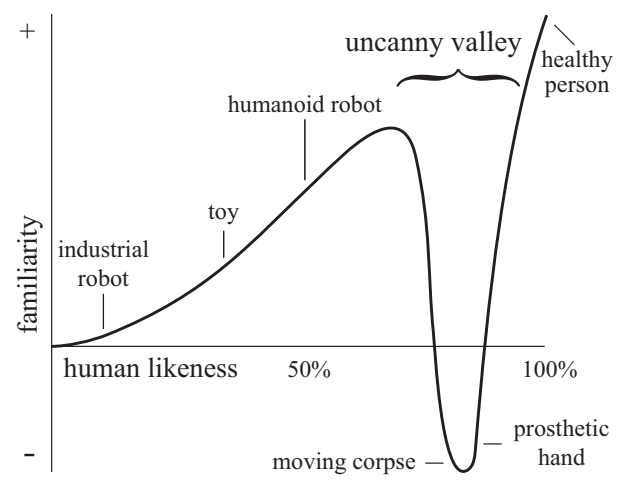

(b) A graphical representation of Mori's Uncanny Valley. [46]

Fig. 3 The problem of eeriness.

30] propose that we can learn from Victorian-age servant-employer relationships in designing robots. Robots should stay out of sight whenever possible, understand complex social contexts, predict employer needs and wants without being presumptuous, etc.. Ideally, these robots can enter homes and form social relationships which people can relate to, an approach less intrusive than forcing the domestic environment to change in order to fit the robot's particulars.

Design methodology, such as appearance, actions, and behavior, will have a large affect on how people perceive domestic robots and the condition of owning one. In particular, the communication paradigm the robot design employs to understand and fit into social contexts will be a crucial component in the robot's chances of acceptance into an application environment.

\subsection{Domestic Robots}

The general population, arguably, has a practical understanding of what a robot is, but most people would have difficulty coming up with a clear definition. 
Roboticists generally resort to domain-specific definitions or simply rely on common sense understanding, where robots are often described as machines that have intelligent behavior, resemble (physically and behaviorally) a human or animal, are mobile, are able to physically interact with their environment, and so on.

Robot is a term currently subject to a large degree of interpretative flexibility, its meaning depending upon context, the people interacting with the robot and the task at hand, rather than according to some universal meaning [49]. The social understanding of a robot has not yet reached a consensus. While originally robot meant an artificial worker [14], since then development in industrial applications and general automation, science fiction media, as well as science-fiction-inspired advanced research has muddled and diversified the meaning. For example, while a toy company may sell an electric, walking toy as a robot, others may argue that it is not a robot due to the lack of intelligence.

Fleck [22] predicted a movement away from the universal robot toward application-specific robots, and argued that social understanding of robot will similarly move toward specific domains and usages. This is expected to lead toward closure, providing a clear distinction between robots based on categories such as task, operation setting, and level of autonomy, e.g., industrial, military, and domestic robots.

It is yet not clear, then, how domestic users on a large scale will respond to robots that enter their personal spaces, and how this interpretation will relate to human perception of other kinds of robots (e.g., military robots). Will domestic robots be seen as just another electronic appliance along with the microwave and home theater system? Will people relate more strongly to science-fiction-inspired concepts of domestic robots? Or will domestic robots trigger a new and unique response?

We propose that users will perceive domestic robots as a new kind of entity. For the purposes of this paper, we define a domestic robot to be a machine that (a) is designed to work with individuals and groups in their personal and public spaces, (b) has a dynamic spatial presence in those spaces, and (c) can "intelligently" interpret its environment and interact physically with it. A robot can alter its presence and influence its surroundings by moving itself or altering its morphology (such as turning its head or moving its arms). We recognize that "intelligence" in this context is likewise defined according to very subjective criteria, a factor that complicates the issue and inhibits the simple, hard-andfast categorization of domestic robots. Our definition does not require robots to resemble humans, to be mobile, or to communicate using natural language.

\subsubsection{Instances of Domestic Robots}

As a basis for discussion in this paper, we focus upon just two cases of domestic robots, one representing a practical product that has already been commercialized, and the other a more futuristic design that is still at the pre-commercialization stage.

The iRobot Roomba [32] (see Figure 4(a)) is an autonomous and mobile vacuum cleaner robot that is affordable, has effective utility, and is a commercially successful product. The Roomba has been introduced into existing home environments, with the overall product (design, implementation, etc.) being sensitive to existing in-home cultures and routines [23]. The Roomba, however, is a utility robot which is meant to independently do its task while staying out of people's way. Its design is such that a user can simply push a start button and walk away; thus it adheres to many characteristics of the traditional servant (as in $[29,30]$ ).

The second robot is the RIKEN RI-MAN [50] (see Figure 4(b)), a personal assistant robot currently under development. RI-MAN is designed to lift people who need assistance and to carry them around their homes. The RI-MAN can dramatically improve the quality of life for the people it helps, and lower their dependence on other individuals. Unlike the Roomba, which works by itself (to clean floors) and stays out of the way, the RI-MAN is designed to directly work with humans, its users being the most crucial component of its design

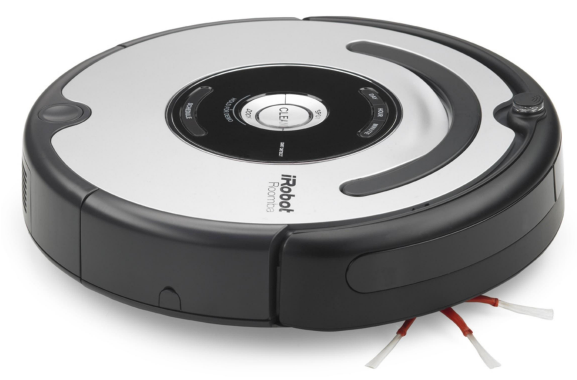

(a) iRobot Roomba. [32]

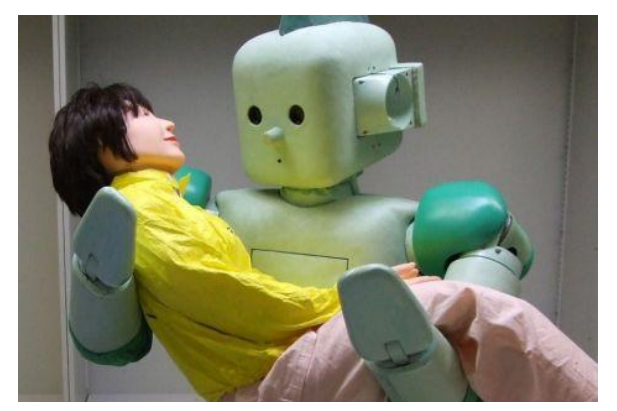

(b) RIKEN RI-MAN. [50]

Fig. 4 The two domestic robots used in our analysis. 
space. This introduces unique questions such as how robots like the RI-MAN will relate to personal space and privacy, as well as a larger trust concern. The RIMAN can physically hurt people, or can cause problems by failing to perform as required, for example, by not carrying them properly from one location to another.

\section{Observations in Social Psychology, a Survey}

We now present four social-psychology behavioral and decision-making models that have been applied to the adoption of technology but have not before been explicitly applied to robots. We consider these theories from a human-robot interaction perspective with the intent of gaining insight into factors that affect how people see domestic robots. Rather than presenting each model in full detail, we distill each into simple representations and outline their primary focus, considerations, and perspectives as a way of bringing to light different ways to analyze technology in social contexts.

The Theory of Reasoned Action (TRA) [2] assumes that rather than being controlled by capricious subconscious forces people are generally rational and leverage information available to them. TRA bases this on observations of both "attitudinal" (i.e., personal) and "normative" (i. e., social) beliefs. Applied to the problem of technology adoption or non-adoption, the attitudinal concerns include opinions of utility, efficiency gains, and how a technology fits into a given lifestyle. The normative beliefs include social views, pressures, expectations, and reactions to adopting a technology. Perceptions are more important than actual outcomes, and perceptions of outcomes can be more important than the perceptions of the robots themselves. A person may acquire a robot simply because they believe it will have a positive impact (e.g., creating more free time), even if there is little or no actual evidence that it will do so [1]. As key to shaping these beliefs, TRA points to lifetime experiences, and past actions and events. Sometimes beliefs are inferred from other knowledge, some beliefs being dynamic and others static [2].

The Theory of Planned Behavior (TPB), an extension to TRA, adds an explicit focus on perceived behavioral control and points more to external factors (media, social acceptance, etc. [40]) than to "previous experience" in the TRA model. This focus tries to accommodate the rapid change and perceived complexity of technology, where previous experience may be lacking and users are wary of difficulty of use. A third model, the Technology Acceptance Model (TAM) [19], is specifically designed to explain and predict computer use, behavior, and adoption. TAM lacks explicit consideration of social and normative variables and focuses on the perceived ease of use and usefulness of computers, based on external variables, as key to how users form attitudes. This emphasis represents a more narrow (but focused) version of TPB's perceived behavioral control.

These models take varying perspectives to unveiling important and unique characteristics of technology adoption [40]. TRA may not handle problems associated with rapidly-changing technologies, while the focused nature of TAM may restrict the scope of its considerations, for example, if social pressure is part of a person's evaluation of a technology's ease of use. TPB would explicitly consider this in the framework from various viewpoints while TAM would simplify by integrating it with other ease of use concerns. However, the more thorough (and wide) nature of TPB may make it difficult to apply meaningfully across various contexts.

The above models primarily take a personal perspective, and are less attentive to the domestic household itself. The Model of Acceptance of Technology in Households (MATH) [62,63], a domestication-oftechnology framework that focuses on the home, was developed around an extensive longitudinal study of the adoption of PCs into over seven hundred households all across America, primarily concerning the factors that people cited for or against adoption. Interestingly, the factors cited for adoption (status and utility gains) did not line up with the factors cited for non-adoption (fear of obsolescence and media influence), and only $45 \%$ of those who claimed they intended to adopt the PC did so six months later, suggesting that fears may strongly overpower perceived gains. While, as we argue, domestic robots are fundamentally different from other technologies, PC-specific models such as TAM and MATH provide perhaps the closest reference framework. That said, we apply these models carefully, considering the robot-specific issues as we do so.

MATH identifies that, in comparison to other contexts, household decisions have a more normative structure and are highly affected by social pressures, views of relevant others, and media [12,63]. This includes the perception of hedonic gains (entertainment, fun), family, friend and social network influence, and perceived barriers or rules surrounding adoption, such as lack of knowledge (inability to properly use a product), prohibitive cost, or regulations requiring/restricting adoption of a technology $[17,63]$. Media influence from secondary sources such as TV and newspapers is particularly strong for early adopters [51] where there are fewer informed friends and families to exert pressure, and the media often provides the first impressions. The hedonic value (pleasure) and social gains derived from a product, through both possession and use, have played a strong role in technology adoption in the past [51], 
being the primary reason for adoption of such things as video games. Adopting a technology also has social gains including public recognition or being a knowledge reference within a social group [64]. From a attitudinal perspective the home has a strong focus on factors such as price, depreciation, maintenance, and space requirements: Venkatesh et.al [63] found that non-adopters primarily cited fears of technology obsolescence.

In the study behind the MATH model, status gains from having a new technology were cited as the primary reason for adoption, with social pressure from family members, hedonic gains, and personal utility cited as contributing reasons. For non-adopters (both "intenders" and "non-intenders"), the social influences and barriers were most significant, with negative influence from secondary sources being the largest factor, for example where due to media representation parents fear for the safety of their children using the internet.

\section{Robots, from a Social Psychology Perspective}

The models above represent unique, and sometimes conflicting perspectives on the domestication of technology. These models provide a base set of tools substantiated by a body of discourse and research that enables us to focus upon exploring how domestic robots fit into the existing understanding of domestication. In this section, we utilize the perspectives and nuances of the above models and apply them directly to the specific challenges of domestic robots.

\subsection{Initial Exposure to Domestic Robots}

The core of the TRA model is that beliefs about a given technology are based on lifetime experience [2]. This is supported by early studies suggesting that the way that robots are introduced to a home (or person) is crucial to the formation of lasting opinions of the technology [23]. Since robots have not yet entered the home on a large scale, perhaps experiences with other technologies will have a strong influence on beliefs thus positively or negatively shaping absorptive capacity. Which previous experiences people will draw on, however, is a function of how the robots themselves and the condition of owning a robot are perceived. Perhaps some robots will be seen as just another home appliance much like PCs, TVs, and personal music players, in which case people would draw upon their experiences with these devices in order to understand domestic robots. However, if robots are perceived as being fundamentally different from other domestic entities then it is not entirely clear which experiences people will draw upon.
Perhaps for sociable robots people will draw from their experiences with children or animals. We argue, however, that robots will fall in between, with people building on past experiences and external sources, inferring new beliefs specific to robots. The image of owning a robot is based on beliefs (not necessarily facts), and so (as MATH points out) media may have a strong influence on shaping these beliefs. This is particularly true for earlier adopters who have less to go on, and may be amplified by the unique nature of robots. Perhaps the strong role of media and exposure to science-fiction has prepared people and has conditioned Pavlovian responses [48] to domestic robots, such as fear of large robots or the attraction of cute, small robots.

TRA points to the utility, effectiveness, and price of robots. While we can expect the trend of utility gains from technology to be continued by robots, people must also perceive them as having a useful purpose. Recent findings [23] suggest that people without prior experience are not always ready to believe that robots are effective, hinting that other attitudinal factors may have to initially play a larger role in the way robots are perceived. However, utility may not be as key as it seems. Venkatesh et al. [62] found in their study that people who intended to adopt a PC cited utility as the motivation twice as often as adopters did in retrospect, suggesting that utility may be an excuse used as a rationalization when other factors (e.g., social status, being a knowledge reference) are the real motivation.

Regarding price, a large part of the Roomba's success could be that it is in the same price range as a regular vacuum cleaner. More advanced and expensive robots such as the RI-MAN will likewise need to create their own balance between price and quality of service, in consideration of the available non-robotic alternatives.

Concerning normative beliefs, MATH [62] suggests that, following the technological trend, there will be social status gains or expectations associated with owning the newest technologies (including domestic robots) that may persuade people to adopt. Social pressures can also be manifested through concerned family members (such as children encouraging parents to adopt automatic vacuum cleaners [23]), a point which may be very influential given current concerns surrounding aging populations in western countries and Japan. However, some people are embarrassed by such automation technology, in that they are afraid to appear lazy to their peers. The Roomba is small enough to store in a closet and the nature of its work (i. e., it does the same task as a regular vacuum cleaner) makes it easy for an owner to conceal the fact that they have one, if they so wish. On the other hand, the Roomba has been de- 
signed and marketed as a stylish household appliance, which may help overcome some of these concerns.

Conversely, the the shear size and mass of the RIMAN, as well as the nature of its work, makes it very difficult to conceal. This problem, however, may be short lived if adoption becomes more common. In the RI-MAN case, the necessity of assistance may overcome such concerns, similar to canes and wheelchairs for people who experience a loss of mobility.

Venkatesh and Brown [63] found the "obsolescence of technology" to be a very large factor for PC adoption, although it is not clear how these concerns will map to the domestic robot. Conceivably, a robot is purchased for a particular purpose and will continue being useful until it breaks. This differs from the PC which, as software demands increase, can no longer execute software and perform the same basic tasks for which it was purchased (such as sharing documents, checking email, etc.) long before it physically breaks. Perhaps, then, robots will only be replaced when newer models offer a very large gain in capabilities and applications to new tasks. The hardware/software model of robots may lay between the PC and traditional appliances that are generally not replaced until they break. Regardless, the resulting architecture will have a very large impact on the adoption of domestic robots.

MATH also points to a normative focus on perceived real barriers, including possible legislation controlling the use of a robot or lack of facilities in the home to deal with a robot. Currently, as robots are not yet controlled by law and use standard household infrastructure (electrical outlets and internet connections) this does not seem to be an issue. However, we can expect legislation to emerge with the proliferation of robots for such things as confining their use and controlling their collateral impact (e.g., your lawnmower robot damaging the neighbors flowers).

\subsection{Control and Safety Regarding Domestic Robots}

The TPB model points to the importance of perceived behavioral control in forming opinions about technology [40] such as users believing they can control when and how technology operates, how adopting such a technology affects their social status, and all other factors of concern. TAM narrows these criteria and places emphasis on the perceived ease of use.

Which of the two emphases is more accurate, behavioral control or ease of use, is subjective to the people involved and the nature of the particular robot. In either case, an important factor is the intersection of a person's skill set and the perception of the skills required to operate a robot. Given that early adopters tend to be better educated [51,63], perhaps educated people have more confidence or skills around advanced technology. However, this may be less of a factor for domestic robots as there may not be many skills transferable from other technologies such as the PC, or from other contexts such as the workplace.

Although the ability to control a robot is always important, the key issue for social robotics becomes one of personal safety. Despite safety tests and assurance by designers, the autonomous and physical presence gives the robot a "life of its own" and can override user perceptions of control. Just as with animals (or people), this fear will be a function of robot capability, size, and will be heavily influenced by experience. For example, similar to the Roomba, most people are not worried about a small kitten or a puppy as they feel they can control the animal if it gets out of hand. With larger animals, such as an untrained large dog, a cougar or a wild horse, this confidence is more difficult (or impossible) to achieve. Even with smaller animals (or robots), capabilities are key: approaching a wild and panicking adult cat is a very scary venture as we know the cat has teeth and very sharp claws. The Roomba, however, has no claws, and is unable to hurt us as long as we keep our fingers away from the cleaning mechanism (a danger we are familiar with when using a regular vacuum cleaner), and so we feel safe around it. On the other hand, the RI-MAN is like a large trained animal: we can learn to trust it, but are still worried about what will happen if it breaks its training (programming).

The Roomba is marketed as a simple "clean with the touch of a button" device, a successful strategy where it only does a single task and only when commanded. Furthermore, its small size and harmless capabilities means it is easy to move or disable and the user can establish virtual walls which restrict the Roomba to a particular room or region. Regardless, people are worried about the Roomba bumping into furniture or knocking down breakables [23].

The RI-MAN may have more difficulty with control issues as it does complex tasks that involve performance ambiguity and its physical size and weight make it impractical for an average person (let alone a needy user) to move or lift it in a dangerous situation. Further, the strength of the robot's arms and its mobility makes the robot quite dangerous in a worst-case malfunction scenario. It is to be expected, then, that the damage-to-furniture type of concerns voiced regarding the Roomba will be dramatically amplified in the RIMAN case. Until robots and artificial intelligence algorithms prove themselves to users, it is expected that this doubtful and wary approach will be a strong factor in peoples' considerations. 


\subsection{Having Fun}

MATH gives explicit consideration to hedonic gains, which have been shown to have shaped other technologies such as the PC in the past [51]. While the Roomba and RI-MAN do not directly address hedonic needs, they may do so indirectly: the Roomba saves time while the RI-MAN increases a person's mobility. Further, some robots are for aesthetic purposes only, much like dynamic art, such as the SONY Rolly [58], which moves and dances while playing music.

The robotic toy is yet another recreational application. Devices like remote-controlled cars have long been marketed as "robots" even though they do not fit our definition in this paper. More recently, however, more genuine advanced robotics have been marketed to consumers in the form of toys. The prime example of this is the Sony AIBO robotic dog, a toy which can move around, sit, play with a ball or bone, take pictures and send them to your email, and even has a complex behavioral and artificial intelligence model to mimic a real puppy (Figure 5). Despite this, however, the AIBO was not commercially successful, and Sony stopped production. The exact reason for the toy's failure is not clear, but it is likely related to the price and the dog's lack of movement capabilities. It sold for over $\$ 2000$ USD, which is a steep price for a toy that has no direct utility or proven history, and it moved very slowly, got stuck easily, and could not traverse stairs. A more successful example is the line of affordable $(\$ 50-\$ 100$ USD) robotic toys from Wowwee [28], including the humanoid Robosapien and a flying robot called Dragonfly. These examples, however, are not really robots under the definition proposed in this paper: the Dragonfly is completely remote controlled, and the other models only have simple abilities and weak interpretation of their environment. Some of Wowwee's more advanced models, such as the Robopanda, are still extremely limited in their abilities. Because of this, these robots enter homes in much the same fashion as a remote-controlled car or battery-powered doll might. They have a modern feeling of novelty but to the average consumer they are still single-purpose toys that fit the existing play paradigms in the home. This contrasts strongly with video games, the internet, the $\mathrm{PC}$, and television, which each provide a fundamentally different dimension to the world of domestic fun.

As robotic toys become more capable we may see a similar thing happen. For example, a Robosapien-likerobot that has just enough awareness of its surroundings to naively follow its owner and play simple games, help them fold the laundry, or even tell a few jokes, would be well beyond any toy available today. To many

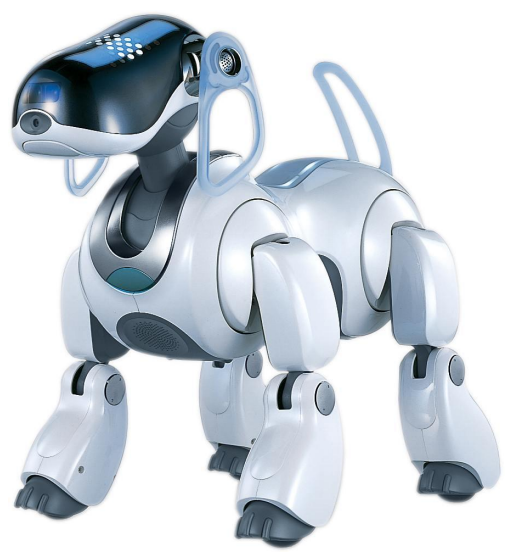

Fig. 5 The Sony AIBO Robotic Dog.

people, such a toy could become a kind of simple pet or companion, and would enable a whole new range of play possibilities not previously possible (as Isaac Asimov's short story Robbie [4] explores). With this in mind, then, presenting robots as toys may help to overcome understanding and acceptance barriers allowing people to categorize these new entities effectively and easily.

One type of emerging robot is the personal sexservice robot. Various producers around the world are working on such products (such as AndyDroid [3], Figure 6). These will no-doubt be successful given the existing markets for a sexual devices including realistic dolls. The interesting question, however, is what will happen when these sex robots become increasingly capable of interpreting, understanding, and intelligently interacting within their environment. How far will the human mind allow the anthropomorphism of machines to go? Will people fall in love with their robots? How much jealousy will people feel if their partner decides to have sex with a robot? [37].

If these become successful, even within a minority of people, such personal experience with a robot may be a key component of the acceptance of robotic technologies. Someone who feels they have an understand-

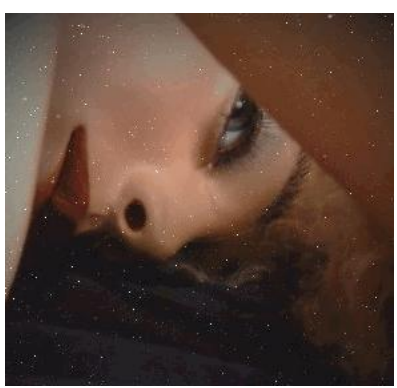

(a) Andy, a female sex robot. [3]

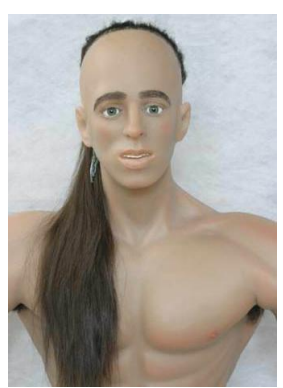

(b) Nax, a male sex robot. [3]
Fig. 6 Two sex robots produced by AndyDroid. [3] 
ing and trust of robots through their experiences in the bedroom might be more willing to bring alternate models in to clean, cook, or play with their children.

The idea of robotic companionship and friendship is a strong one. Given sociable robots, and the fact that people already anthropomorphize robots with humanlike characteristics, it will be no surprise if people start to feel an attachment to them as already happens with material things such as sports cars, collectible items, teddy bears, or various other items that are important for personal reasons. Given the uniqueness and active role of robots, these kinds of bonds may perhaps become stronger and move closer to the kinds of bonds experienced between two people.

Particularly for robots such as the RI-MAN which has a human-like appearance, replaces a traditional human role, and provides a service that may result in a feeling of gratitude and perhaps emotional attachment from the owner, the development of a sense of companionship would be an almost-natural progression. This has happened, for example, in military settings [26]: an Army colonel canceled a mine-sweeping robot experiment as the robot was getting mutilated, stating that the test was inhumane, soldiers awarded robots battlefield promotions, and in one instance demanded that a damaged robot (in this case an iRobot Packbot [33], Figure 7, named Scooby-Doo) be repaired instead of replaced at a fraction of the cost.

As intriguing as the idea of robotic companionship may seem, however, it is doubtful that this idea will have any initial impact on intention to adopt beyond what existing toys and electronics already offer. Robots are currently very limited, and people will likely not consider the deeper reaches of the companionship factor until there is experience and a cultural understanding

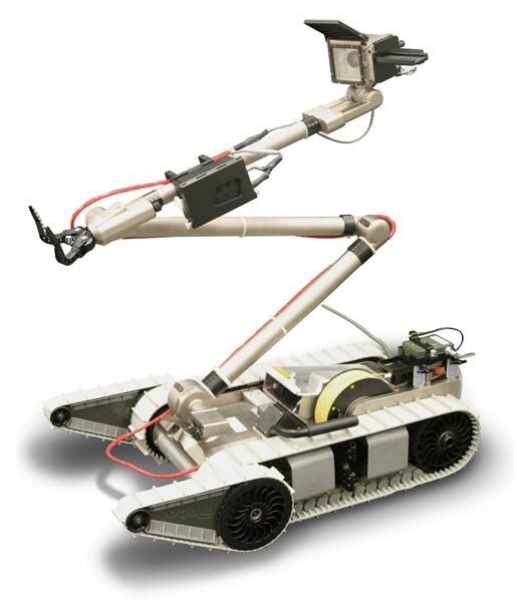

Fig. 7 The iRobot Packbot. [33] of such a phenomena. Initially, at least, companionship may just be a secondary product of purchasing a robot.

\subsection{The Role of Social Intelligence in Design}

The social psychology models presented in our survey (Section 3) are limited in that they do not consider issues of what we could call "social" intelligence, referring to the ability of technologies to sense the dynamics of social environments and to fit in to them. Social intelligence has not been a major concern for traditional, simpler domestic technologies, most of which have limited capabilities to act independently of specific user input. There are more invasive domestic technologies such as the emerging intelligent home environment that can sense changes in the physical environment and act accordingly (for example, operating lights, temperature and climate controls). Robots have similar capabilities, but also intervene in a unique way in social environments, thus heightening the requirement for designers to consider the social intelligence issue.

The Roomba, although successful, is not explicitly designed to follow sociable robotics principles. It has a mechanical appearance and utilizes simple blinking lights for status messages, although the sounds it makes can be construed as happy or sad, and the newest models have a synthetic-speech introduction. Despite its extremely basic interaction design, however, people anthropomorphize and zoomorphize the Roomba anyway, giving it human and social characteristics [23]. In particular, its movements - although mechanical - are described using words such as cute or pathetic. Many people give their Roomba a name and talk to it while it cleans [23]. This suggests that in addition to being functionally useful, the Roomba can become a social part of the home and in a sense, a social participant in the family, not that different from, say, a pet hamster. But as it appears that humans are quite willing to anthropomorphize or zoomorphize objects that have very minimal communication abilities (consisting only of simple movements), such familiarity may be obtainable to a limited degree using design methodologies other than social robotics. However, it is also quite possible that the Roomba exhibits social principles (such as movement patterns) that are not-yet clearly defined or understood.

The RIKEN RI-MAN, on the other hand, is explicitly designed to have a human-like appearance: it has a human head, face and arms, soft skin, ears that listen and a mouth that speaks, and social programming that allows it to follow communication protocols such as gaze during conversation. It remains to be seen how this robot is received by general users, but this 
level of human resemblance puts the robot into risk of eeriness problems. Currently, the Roomba's approach shows promise for avoiding this while still having a level of social interaction. We think that successful domestic robotic interfaces will have to be somewhere in between the Roomba and the RI-MAN, where the robots offer enough cues for in-depth social interaction but are still perceived as a mechanical entity.

It is not yet understood how higher-level social savvy in a robot, such as the ability to fit into the social and activity structures of the home, will affect the perception of the robot. Technologies such as the PC and the Roomba that offer no explicit model to interpret the social environment suggest that active social understanding is not necessarily required for a technology to be successful in a domestic setting. The problem with this claim, however, is the simplicity of how these socially-ignorant technologies interact with physical environments. The goals of more advanced machines such as the RI-MAN require them to actively interact in spaces shared with people, and to navigate environments with a large degree of autonomy and intelligence. They should have an understanding of what people are doing (such as a sleeping baby, a person using the washroom, or a child doing homework) and alter its actions appropriately, with a calculated impact on the social fabric of the home.

We argue that social savvy is a secondary concern that is directly coupled with capability: the more intelligent and capable a robot is, the more people expect from it on all levels, including social understanding. This does not mean, however, that people are unhappy or impatient with unintelligent technologies, but suggests that robot designers may sometimes want to lower the intelligence, or appearance of intelligence, of their robots in order to lower the social expectations of users. Perhaps people will be forgiving and will accommodate them in much the same way they do with pets or children, finding it simply natural that the robot does not understand. For example, a dog is taught not to bite or bark excessively as people know dogs can learn this, but fish are not trained in the same fashion: rather, signs that say "do not touch" are affixed instead. Similarly, parents simply apologize when infants pull other people's hair, but when the infant becomes a toddler they are (usually) scolded and instructed not to do so. While people may rationalize when the vacuum-cleaning robot interrupts their dinner, this comparison will likely break down for critical and dangerous scenarios, and people may have zero tolerance for domestic robots that break plates or flood the floor while cleaning. Following, in respect to using the model of the Victorian servant for robotic interface design [30], it may not be necessary to take this metaphor to very complex levels of social understanding.

\section{Guidelines for Considering the Acceptance of Domestic Robots}

By looking to social psychology we have gained various perspectives on the dynamics of how technology has been domesticated in the past. In the previous section, we explored how the ideas put forward by various models relate to the unique issues and concerns surrounding robots. In this section, we distill our discussion and findings into a set of guidelines, an intersection of the models presented and the specifics of robots. We present these in a form that we hope designers of domestic robots and their interfaces can use as high-level guidelines.

The are two sets of guidelines: first, we discuss factors that we feel influence how domestic users perceive domestic robots, and second we outline which influences are key to forming these perceptions.

\subsection{Factors Affecting Acceptance}

Several guidelines can be used to reconceptualize a robot-design problem and to analyze robotic interfaces in terms of how people will accept them. It is the perception of these points, in contrast to some measurable value, that is important.

Safety - Robots have an autonomous physical presence that, in a worst-case-scenario, can damage household objects or seriously injure and kill people. Robots provide a level of potential danger seldom experienced with other domestic technologies in the past, and so this concern may be disproportionately important and may overshadow any and all other gains and benefits.

Accessibility and usability - The capabilities and complexity of robots raises serious accessibility concerns. Existing technology fears such as lack of knowledge, usability, and behavioral control (already shown to have been a problem for PC adoption), will escalate given the physical presence and dangers of robots. Other barriers include facilities and space requirements within the home, financial practicality (affordability, maintenance and obsolescence) and legal barriers and regulations.

Practical benefits - People really care about the utility gains promised by robots, and the potential impact on their quality of life. Robots must not only be useful, but need to fit properly into the social structures of a given particular lifestyle. At the same time, however, 
people may be overly dubious about the capabilities of robots.

Fun - Direct fun (and secondary gains such as more free time due to utility gains) is a very important consideration for domestic procurement decisions. Robot designers have already recognized this and have introduced robotic technology satisfying this need. Further, companionship and comfort are basic human needs that robots may be able to meet. Perhaps, similar to the way games help drive PC technology, entertainment robots may serve as a catalyst for the entire domain.

Social pressures - Conflicting social pressures should be expected concerning domestic robots. As robots become common we can expect the emergence of social pressures that will motivate adoption, for example the motivation for a family to appear to be "modern". On the other hand, negative pressures such as appearing lazy or wasteful can also be expected.

Status gains - Being perceived as a cutting-edge person or family, or being recognized as a knowlegable reference by neighbors or co-workers, has been important for adoption of technologies in the past. It is unclear how this will relate to domestic robots, but science fiction and research hype has arguably created a fairly positive and luxurious image of robots for people to consider. Designers can take advantage of this image.

Social intelligence - There is a tendency for people to anthropomorphize robots more than traditional domestic technologies, meaning that an expectation of social intelligence may inherently result from a robot's design. This can be leveraged by portraying robots as being easy to communicate with, but can also lead to disappointed users when expectations are not met.

\subsection{The Perception of Factors Affecting Acceptance}

As stressed throughout this paper, perception of factors is as meaningful or often even more meaningful to domestic users than the actual facts. Here we outline some of the key sources and points that influence how people shape their understandings and perceptions of the factors presented in Section 5.1.

Previous experience - Being a primary source, these include personally-experienced lifetime actions and events as well as personally inferred beliefs, with education and initial exposure being a large component of this factor. Previous experience with animals and children may be influential here as well. Given the new and unique nature of robots, a robot can be designed to influence users to draw on particular past experiences as desired by the designer.

Media - People's previous experience with robotic technologies is very limited, so we expect them to strongly leverage media as an important source of information. This includes classic science-fiction-like literature, movies, and television, as well as more modern and fact-oriented news sources. Designing robots around media trends can be an important aspect of acceptance.

Personal social network - Opinions and perspectives offered by friends, neighbors and family have a large influence on how people perceive robots. Although robots are new and as such the social network itself will be less informed, this will likely be an important factor nonetheless. Although it is not clear how designers can influence this factor, somehow making a robot conducive to socializing would be helpful here.

Robot design methodology - The design of a technology, its physical appearance, actions, interface, and all other aspects of design, directly influence which previous experiences people use when forming their understanding of a given entity. To a large degree, this constitutes a consumer epistemology of previous knowledge and experience upon which consumers may develop absorptive capacity as discussed above. With robots, designers can either leverage or constrain user tendencies to anthropomorphize. Designers may also use the robot's dynamic physical presence as means to influence perception, for example, by limiting speed or agility in an attempt to convey a harmless or safe robot. Robots can use human social interaction (gaze, facial expression, physical proximity) in new ways that other, previous technologies are unable to do. Moreover, eeriness phenomena may also be useful in the design of social robots, for example in order to make domestic users aware of potentially dangerous robots or situations.

\section{Conclusion}

Social psychology offers an in-depth understanding of how people perceive technologies and how they construct those perceptions. In this paper, we discussed several of these social models and applied their concepts in order to high-light many of the specific social factors that are likely to affect the adoption process for domestic robots. Our analysis is by no-means complete, and we expect it to evolve as the technical characteristics and social meanings of the domestic robot evolve [35].

We hope that this paper will provide practitioners with some new perspectives on the design of social robots in domestic settings and that it will make the design community more aware of theoretical tools that can be drawn from the social sciences and applied to the exploration and analysis of domestic robots and robotic interfaces. 


\section{Acknowledgments}

Our research was supported by the National Sciences and Engineering Research Council of Canada (NSERC), the Alberta Informatics Circle of Research Excellence (iCore), the Japan Science and Technology Agency (JST), and various University of Calgary grants. We would like to thank Maria Bakardjieva for the initial inspiration for this work, as well as members of the University of Calgary Interactions Lab and the JST ERATO Design UI group for help and support.

\section{References}

1. Ajzen, I.: The theory of planned behavior. Organizational Behavior and Human Decision Processes 50, 179-211 (1991)

2. Ajzen, I., Fishbein, M.: Understanding Attitudes and Predicting Social Behavior. Prentice Hall, Englewood Cliffs, NJ (1980)

3. AndyDroid: AndyDroid. WWW, http://www.andydroid .com/index2_eng.htm (2008)

4. Asimov, I.: I, Robot. Grafton Books, London (1968). A collection of short stories originally published between 1940 and 1950

5. Bartneck, C., van der Hoek, M., Mubin, O., Mahmud, A.A.: "Daisy, Daisy, give me your answer do!": switching off a robot. In: Proceedings of the 2nd ACM SIGCHI/SIGART Conference on Human-Robot Interaction, 2007. HRI '07, Washington, D.C., USA, March 10-12, 2007, pp. 217-222. ACM, ACM Press, New York, NY (2007). DOI 10.1145/ 1228716.1228746

6. Bartneck, C., Verbunt, M., Mubin, O., Mahmud, A.A.: To kill a mockingbird robot. In: Proceedings of the 2nd ACM SIGCHI/SIGART Conference on Human-Robot Interaction, 2007. HRI '07, Washington, D.C., USA, March 10-12, 2007, pp. 81-87. ACM, ACM Press, New York, NY (2007). DOI 10.1145/1228716.1228728

7. Bijker, W.: Do not despair: there is life after constructivism. Science, Technology and Human Values 18(4), 113138 (1993)

8. Breazeal, C.: Affective interaction between humans and robots. In: Proceedigns of the 6th European Conference on Advances in Artificial Life, 2001. ECAL '01, Prague, Czech Republic, September 10-14, 2001, pp. 582-291. Springer science \& business media, Springer Berlin / Heidelberg, London (2001)

9. Breazeal, C.L.: Designing Sociable Robots. The MIT Press, Cambridge, Massachusetts (2002)

10. Breazeal, C.L.: Emotion and sociable humanoid robots. International Journal of Human-Computer Studies (IJHCS) 59(1-2), 119-155 (2003). DOI 10.1016/S1071-5819(03) 00018-1

11. Brenton, H., Gilles, M., Ballin, D., Chatting, D.: The uncanny valley: does it exist? In: 19th British HCI Group Annual Conference: workshop on human-animated character interaction, Edinburgh, Sep 2005 (2005)

12. Burnkrant, R., Cousineau, A.: Informational and normative social influence in buyer behavior. Journal of Consumer Research pp. 206-215 (1975)

13. Callon, M.: Society in the making: The study of technology as a tool for sociological analysis. In: W.E. Bikjer, T.P. Hughes, T. Pinch (eds.) The Social Construction of Technological Systems, pp. 17-50. Cambridge: MIT Press, Cambridge, Massachusettes (1987)
14. Capek, K.: Rossum's Universal Robots. Pocket Books, New York, NY (1970). Originally appeared as play, 1920

15. Clark, J., McLoughlin, I., Rose, H., King, R.: The Process of Technological Change: New Technology and Social Choice in the Workplace. Cambridge University Press, Cambridge, UK (1988)

16. Cohen, W.M., Levinthal, D.A.: Absorptive capacity: a new perspective on learning and innovation. Administrative Science Quarterly pp. 128-152 (1990)

17. Compeau, D., Higgins, C.A.: Applications of social cognitive theory to training for computer skills. Information Systems Research 6(2), 118-143 (1995)

18. Cornwall, J.: Modern Capitalism: its Growth and Transformation. Martin Robertson, London (1977)

19. Davis, F.D.: A technology acceptance model for empirically testing new end-user information systems: Theory and results. Ph.D. thesis, Sloan School of Management, Massachusetts Institute of Technology (1986)

20. Dholakia, R.R.: Gender and it in the household: Evolving patterns of internet use in the united states. The Information Society 22(4), 231-240 (2006)

21. Dosi, G.: Technological paradigms and technological trajectories: A suggested interpretation of the determinants and directions of technical change. Research Policy 11(3), 147162 (1982)

22. Fleck, J.: The adoption of robots in industry. Physics in Technology 15(1), 4-11 (1984)

23. Forlizzi, J., DiSalvo, C.: Service robots in the domestic environment: a study of the roomba vacuum in the home. In: Proceedings of the 1st ACM SIGCHI/SIGART Conference on Human-Robot Interaction, 2006. HRI '06, Salt Lake City, USA, March 2-4, 2006, pp. 258-256. ACM Press, New York, NY (2006)

24. Friedman, B., Peter H. Kahn, J., Hagman, J.: Hardware companions? - what online AIBO discussion forums reveal about the human-robotic relationship. In: ACM Conference on Human Factors in Computing Sysems, 2003. CHI '03, Fort Lauderdale, USA, April 5-10, 2003, pp. 273-280. ACM, ACM Press, New York, NY (2003). DOI 10.1145/642611.642660

25. Fussell, S.R., Kiesler, S., Setlock, L.D., Yew, V.: How people anthropomorphize robots. In: Proceedings of the 3rd ACM SIGCHI/SIGART Conference on Human-Robot Interaction, 2008. HRI '08, Amsterdam, The Netherlands, March 12-15, 2008, pp. 145-152. ACM, ACM Press, New York, NY (2008). DOI 10.1145/1349822.1349842

26. Garreau, J.: Bots on the ground. Washington Post, WWW, http://www.washingtonpost.com/wp-dyn/content /article/2007/05/05/AR2007050501009_pf.html, Visited April 9th, 2008 (2007)

27. Geller, T.: Overcoming the uncanny valley. IEEE Computer Graphics and Applications 28, 11-17 (2008)

28. WowWee Group Limited: WowWee. WWW, http://www . wowwee.com/, Visited June 11th, 2008 (2008)

29. Hamill, L.: Controlling smart devices in the home. The Information Society 22(4), 241-249 (2006)

30. Hamill, L., Harper, R.: Talking intelligence: a historical and conceptual exploration of speech-based human-machine interaction in smart homes. In: Proceedings of the 1st International Symposium on Intelligent Environments, ISIE, Apr 5-7, Cambridge, UK, 2006, pp. 121-128. Microsoft Research, MSR, Cambridge, UK (2006)

31. Hanson, D., Olney, A., Pereira, I.A., Zielke, M.: Upending the uncanny valley. In: Proceedings of the Twentieth national conference on artificial intelligence, 2005. AAAI '05, Pittsburgh, USA, July 9-13, 2005, pp. 1728-1729. Association for the Advancement of Artificial Intelligence, Association for the Advancement of Artificial Intelligence Press, Menlo Park, USA (2005) 
32. iRobot: Roomba. WWW, http://www.irobot.com /consumer, Visited Feb 9th, 2007 (2007)

33. iRobot: irobot government and industrial robots. WWW, http://www. irobot.com/sp.cfm?pageid=109, Visited May 11th, 2009 (2008)

34. Kiesler, S., Hinds, P.: Introduction to This Special Issue on Human-Robot Interaction. Human-Computer Interaction (HCI) 19(1/2), 1-8 (2004)

35. Kirsh, D.: Explaining artifact evolution. In: presentation at the Knowledge Conference, Irvine, April 22, 2006. University of California, University of California, Irvine, California (2006)

36. Lancaster, K.: A new approach to consumer theory. The Journal of Political Economy 74(2), 132-157 (1966)

37. Levy, D.: Love and Sex with Robots: The Evolution of Human-Robot Relationships. Harper, New York, NY (2007)

38. MacDorman, K.F., Minato, T., Shimada, M., Itakura, S., Cowley, S., Ishiguro, H.: Assessing human likeness by eye contact in an android testbed. In: Proceedings of the 27th Annual Meeting of the Cognitive Science Society, 2005. CogSci '05, Stresa, Italy, July 21-23, 2005. Cognitive Science Society, Lawrence Erlbaum, Mahwah, NJ, USA (2005)

39. Mansfield, E.: The diffusion of industrial robots in japan and the united states. Research Policy 18, 183-192 (1989)

40. Mathieson, K.: Predicting user intentions: Comparing the technology acceptance model with the theory of planned behavior. Information Systems Research 2(3), 173-191 (1991)

41. McMeekin, A., Green, K., Tomlinson, M., Walsh, V.: Innovation by Demand: an Interdisciplinary Approach to the Study of Demand and its Role in Innovation. Edward Elgar, Cheltenham (2002)

42. Michalowski, M.P., Sabanovic, S., Kozima, H.: A dancing robot for rhythmic social interaction. In: Proceedings of the 2nd ACM SIGCHI/SIGART Conference on Human-Robot Interaction, 2007. HRI '07, Washington, D.C., USA, March 10-12, 2007, pp. 89-96. ACM, ACM Press, New York, NY (2007). DOI 10.1145/1228716.1228729

43. Minato, T., Shimada, M., Ishiguro, H., Itakura, S.: Development of an Android for Studying Human-Robot Interaction. In: Proceedings of the Innovations in Applied Artificial Intelligence, Seventeenth International Conference on Industrial and Engineering Applications of Artificial Intelligence and Expert Systems 2004. IEA/AIE '04, Ottawa, Canada, May 17-20, 2004, pp. 424-434. ACM, ACM Press, New York, NY (2004)

44. Minato, T., Yoshikawa, Y., Noda, T., Ikemoto, S., Ishiguro, H., Asada, M.: $\mathrm{Cb}^{2}$ : A child robot with biomimetic body for cognitive developmental robotics. In: Proceedings of the IEEE-RAS/RSJ Intarnational Conference on Humanoid Robots, 2007. Humanoids '07, Pittsburgh, USA, November 29-December 1, 2007. IEEE Computer Society, IEEE Computer Society Press, Los Alamitos, CA, USA (2007)

45. Montalvo, C.: Environmental policy and technological innovation: Why do firms adopt or reject new technologies? Edward Elgar, Cheltenham (2002)

46. Mori, M.: Bukimi no tani: the uncanny valley (in Japanese). Energy 7(4), 33-35 (1970). English translation provided at CogSci '05 workshop: Toward Social Mechanisms of Android Science, Views of the Uncanny Valley. WWW, http://www.androidscience.com/ theuncannyvalley/proceedings2005/uncanny valley.html, Visited Feb 9th, 2007

47. Norman, D.: Emotional design: why we love (or hate) everyday things. Basic Books, New York, USA (2004)

48. Pavlov, I.P.: Conditioned Reflexes: An Investigation of the Physiological Activity of the Cerebral Cortex. Oxford University Press, London (1927). Translated and Edited by G. V. Anrep
49. Pinch, T.J., Bijker, W.E.: The Social Construction of Facts and Artifacts, pp. 17-50. Cambridge: MIT Press, Cambridge, Massachusettes (1987)

50. RIKEN: Bio-Mimetic Control Research Center, RIMAN. WWW, http://www.bmc.riken.jp/ RI-MAN/ index_us.html, Visited Feb 9th, 2007 (2007)

51. Rogers, E.M.: Diffusion of Innovations. Free Press, New York (1995)

52. Schmookler, J.: Invention and Economic Growth. Harvard University Press, Cambridge (1966)

53. Schumpeter, J.A.: Die Theorie der Wirtschaftlichen Entwicklung. Duncker \& Humblot, Leipzig (1912)

54. Scitovsky, T.: The Joyless Economy: an Inquiry into Human Satisfaction and Consumer Dissatisfaction. Oxford University Press, Oxford (1976)

55. Setterfield, M.: The economics of Demand-Lef Growth: Challenging the supply-Side Vision of the Long Run. Edward Elgar, Cheltenham (2002)

56. Silverstone, R.: Beneath the bottom line: households and information and communication technologies in an age of the consumer. PICT Policy Research Papers 17 (1991). Economic and Social Research Council, Swindon

57. Silverstone, R., Morley, D.: Families and their technologies: two ethnographic portraits, pp. 74-83. Futures Publications, London (1990)

58. SONY: Rolly. WWW, http://en.wikipedia.org/wiki/ Rolly _ (Sony), Visited June 30th, 2008 (2008)

59. Steiner, C.J.: A philosophy for innovation: The role of unconventional indivisuals in innovation success. Journal of Product Innovation Management 12, 431-440 (1995)

60. Stoneman, P.: Technological Diffusion and the Computer Revolution: The UK Experience. Cambridge University Press, Cambridge (1976)

61. Swann, G.M.P.: The demand for distinction and the evolution of thh prestige car. Journal of Evolutionary Economics 11, 59-75 (2001)

62. Venkatesh, A.: Introduction to the special issue on "ICT in everyday life: Home and personal environments". The Information Society 22(4), 191-194 (2006)

63. Venkatesh, A., Brown, S.A.: A longitudinal investigation of personal computers in homes: Adoption determinants and emerging challenges. MIS Quarterly 25(1), 71-102 (2001)

64. Venkatesh, V., Davis, F.D.: A theoretical extension of the technology acceptance model: Four longitudinal field studies. Management Science 46(2), 186-204 (2000)

65. Von Hippel, E.: Democratizing Innovation. The MIT Press, Cambridge, MA (2005)

66. Walters, M.L., Syrdal, D.S., Dautenhahn, K., te Boekhorst, R., Koay, K.L.: Avoiding the uncanny valley: robot appearance, personality and consistency of behavior in an attentionseeking home scenario for a robot companion. Autonomous Robots 24(2), 159-178 (2008)

67. Williams, R., Edge, D.: The social shaping of technology. Research Policy 25(6), 865-899 (1996)

68. Winner, L.: Do Artifacts have politics?, pp. 19-39. University of Chicago Press, Chicago (1987)

69. Young, J.E., Xin, M., Sharlin, E.: Robot expressionism through cartooning. In: Proceedings of the 2nd ACM SIGCHI/SIGART Conference on Human-Robot Interaction, 2007. HRI '07, Washington, D.C., USA, March 10-12, 2007, pp. 309-316. ACM Press, New York, NY (2007)

70. Zoonen, L.v.: Gendering the internet. claims, controversies and cultures. European Journal of Communication 17(1), $5-24(2002)$ 